Advanced Computing: An International Journal ( ACIJ ), Vol.3, No.5, September 2012

\title{
Enhancement of Improved Balanced LEACH for Heterogeneous Wireless Sensor Networks
} \author{
Yogesh Kumar $^{1}$ and Kanwaljit Singh ${ }^{2}$ \\ ${ }^{1}$ ECE Department, Guru Nanak Dev Engg. College, Ludhiana \\ kumar_yogesh1087@rediffmail.com \\ ${ }^{2}$ Associate Professor ECE Department, Guru Nanak Dev Engg. College, Ludhiana, India \\ kjitsingh@gndec.ac.in
}

\begin{abstract}
Wireless sensor networks consists of thousands of tiny, low cost, low power and multifunctional sensor nodes where each sensor node has very low battery life. Purpose is to conserve the transmitted energy from various sensor nodes. Various energy efficient algorithms have been designed for this. LEACH uses distributed cluster formation \& randomized rotation of the cluster head to minimize the network energy consumption. Our paper is proposing an algorithm which is the enhancement of existing IB-LEACH. It reduces the energy consumption by using energy bank. This energy bank stores the energy after each round in both routing and clustering phase which overall increases the life time of the network. In this approach, ACTIVE_ROUTE_TIMEOUT is also enhanced by shamming the static parameters of HELLO_INTERVAL, RREQ_RETRIES and NET_DIAMETER. Results are compared through MATLAB and provide better approach than previous ones.
\end{abstract}

\section{Keywords}

Enhanced, IB-LEACH, Clustering, AODV protocol, Simulation

\section{INTRODUCTION}

WSANs consists of small nodes called as sensors, these networks provides three essential functions. Firstly they are used in real time system to monitor physical and environmental conditions such as humidity, temperature, pressure etc. secondly these networks provide reliable and efficient communication via wireless network. Third, these networks are used to monitor and control traffic conditions. These networks can operate on switches and monitor and other devices. Sensors of wireless networks are disturbed in an Adhoc manner. These networks have various algorithms and protocols which is having self-organizing capabilities. These sensor nodes work together to perform various tasks and then gathered information is processed to get required results.

\subsection{Layered Architecture}

Layered architecture of wireless sensor network consists of three layers.

1) Base Station (BS): It is a powerful base station from where other layers arrive.

2) Sensor nodes Layer: Layers of sensor nodes around BS.

3) I Layer: All nodes i-hop away from BS

Base station location is very significant and for multihop networks it is very challenging. Size of sensor nodes can be changed and these changes put a great impact on the wireless sensor 
networks. Various layers are used in this network. Size of sensor nodes puts the effect on resources of the network. Today sensor networks are used in all fields such as Industrial, commercial and other number of applications.

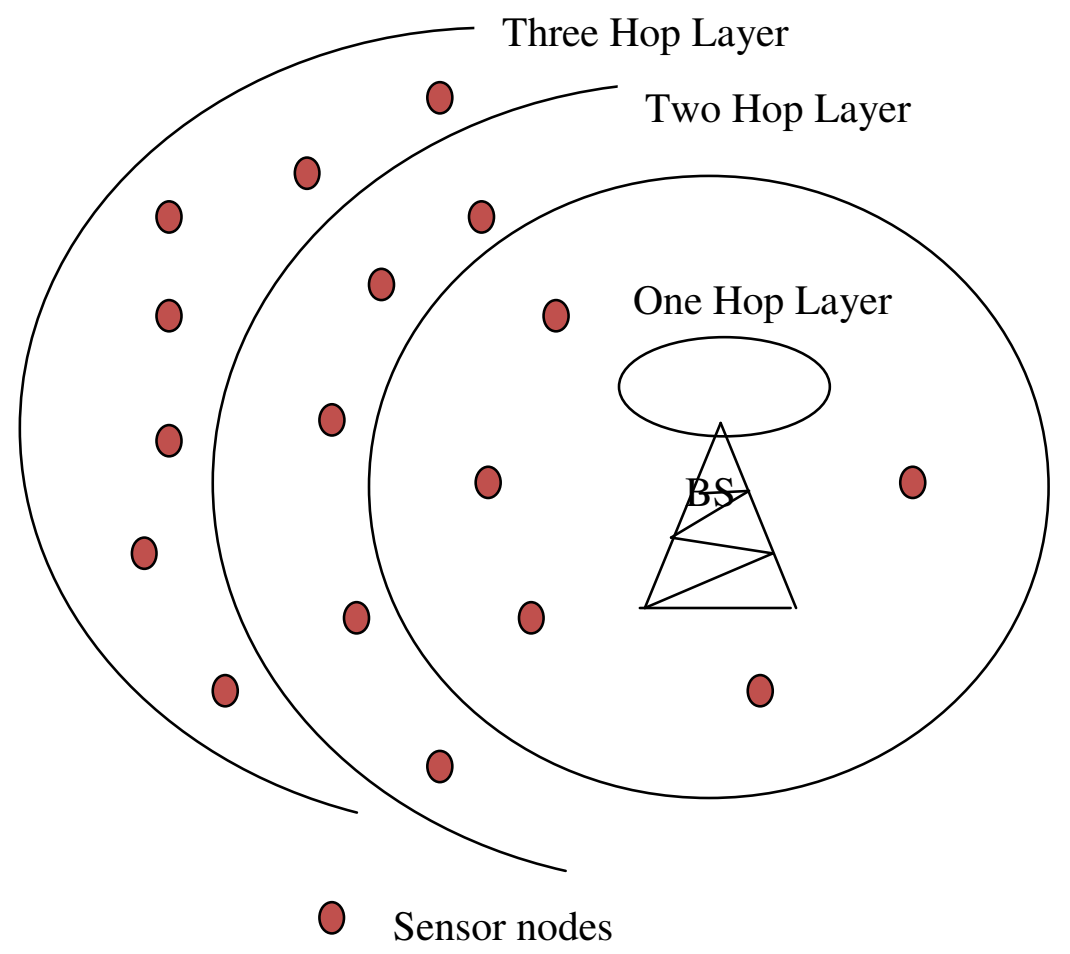

Figure1: Layered Architecture of WSN

\section{ROUTING IN SENSOR NETWORKS}

Routing is a big challenge in wireless sensor networks, because these networks have some specific characteristics. First sensor nodes are power constrained, they have very less energy and it is very difficult to reserve the energy while the nodes are transmitting the bits. Second, these networks are dynamic in nature and must be able to adapt environment changes and requirements automatically. It may be the case that sometime new nodes are inserted in the network and previous nodes are deleted from the network. With this position of the networks may change, which sometimes lead to change in position, energy, tasks and reach ability of the network. Third these networks must be self-configured because of dynamic environment changes. Fourth global IP addressing scheme of wired networks can't be applied to wireless sensor networks. Fifth, transmitted data contains redundant data as multiple sensors can send redundant data at the same time. So many protocols are used to conserve the energy level of the various sensor nodes. Sixth there are so many nodes in the network, so it is very difficult to trace hundreds, thousands nodes of the networks. Seventh, sensor nodes are application specific in nature. So protocols must be designed with care. 
Routing protocol can be divided into two categories. First is flat routing and second is hierarchical routing. These routing depends on the structure of the network. In flat routing, there is no ay hierarchy; all the nodes play some role. In hierarchical routing, clustering method is used in which some nodes are assigned specific functionalities. A cluster is a set of geographically proximal sensor nodes in which one node acts as a cluster head. These cluster heads are further organized into hierarchical levels.

\subsection{AODV Routing Protocol}

This is an On Demand Routing Protocol. In this routes are discovered on the spot. When a sender wants to send the data then this protocol start to sense the nodes. This protocol gives quick response to node or link breakage and if there is any change in the network. As this protocol gives quick response to changing network topology, so this protocol is widely used in wireless sensor networks. This protocol has two stages:

1) Route Discovery

2) Route Maintenance

This protocol discovers the route by sending RREQ (Route Request) and RREP (Route Reply) messages. In establishment phase, it uses HELLO messages and RERR (Route Error) messages. Source sends data to the destination by broadcasting PREQ, with this it finds the destination routes. If the nodes related the destination route has been expired then a fresh node will be inserted immediately and this fresh node having a sequence number which is always greater than RREQ.

Destination route becomes available by sending a unicast message RREP back to the source of the RREQ. RREQ contains broadcast id, source IP address, source sequence number, destination IP address, destination sequence number and hop count. Source IP address and broadcast-id uniquely identifies any RREQ. After receiving the RREP the source node start transmitting the data to the destination.

All the nodes send HELLO messages periodically to each other. So, that if link breakage occurs then nodes will be able to inform each other using RERR (route error) message. This breakage will be informed to source node, after receiving the RERR the source node cancel the route and if desired start to discover new route. New route is discovered again by sending a broadcast route request (RREQ) over the network and destination again gives the reply by unicast the message. The intermediate nodes store this state for a length of time which is given by the parameter ACTIVE-ROUTETIMEOUT. This time is used to send data, if any link breaks or failure occurs then time will be expired and AODV protocol cancels the route and again search for new routes. Default value of ACTIVE-ROUTETIMEOUT is 2 seconds. This is static value which defines how long a route is kept in the routing table after the last transmission of a packet on this route.

This ACTIVE-ROUTE-TIMEOUT value is selected with care, because a small value can lead to a new route discovery even if the route is valid and large value can create a risk of sending the packets on an invalid route. In the first case, the cost is related with new route discovery and in the second case it is the loss of one or more packets. 


\section{PREVIOUS CLUSTERING METHOD OF LEACH}

The Sensor node which is having 2AA battery can last up to three years, this makes routing a challenge in wireless sensor networks. To increase the life time of the sensor nodes, clustering approach is used. Cluster is the combination of various sensor nodes. In this technique cluster head is selected after every round. Residual energy is selected so that equal energy is given to all the nodes.

LEACH is a hierarchical clustering algorithm for sensor networks. It has two phases. One is Cluster set up phase and other is a steady state phase. Each node start with a random number 0 and 1 , if the number is less than threshold $\mathrm{T}(\mathrm{n})$, the node becomes cluster-head for current round.

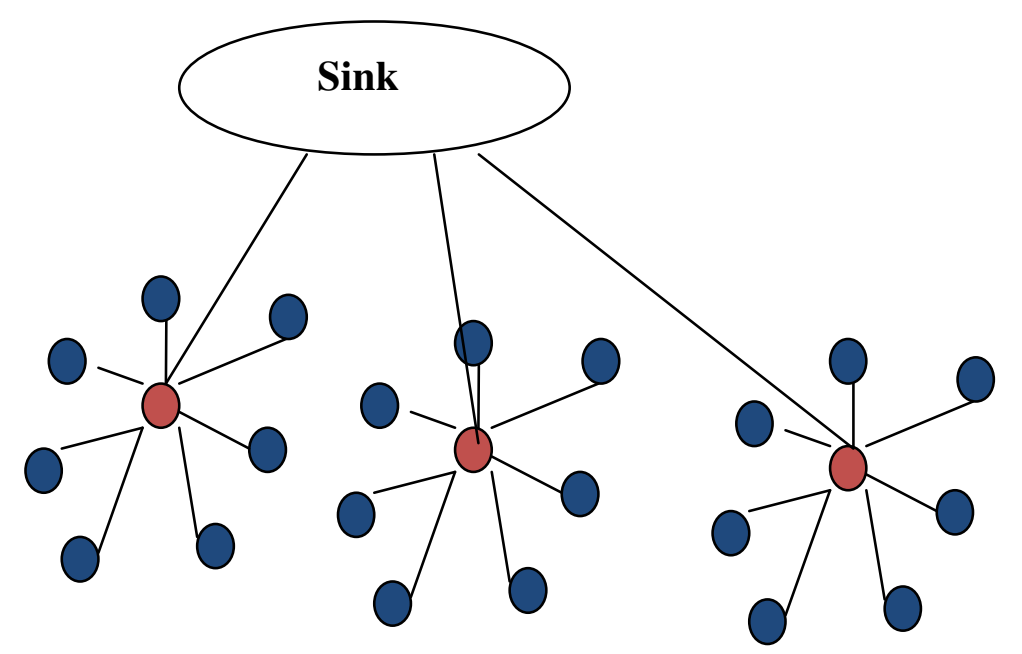

Cluster Head

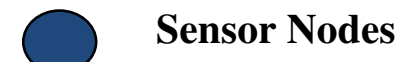

Figure2: Clustering Based on LEACH

Every cluster head sends the data to its own cluster head with in the TDMA time schedule, so that data will be sent timely. It is transmitted in two ways. Firstly, data is transmitted to cluster head nodes in their assigned TDMA slot and in second step the data aggregation take place from cluster head to sink. The frame time for this purpose is defined as follows:

Frame Time $=(\mathrm{CAT}+\mathrm{S}) \mathrm{X}$ Ts

CAT - It is the number of time slots for data aggregation and transmission from cluster head to Base station.

$\mathrm{S}$ - Number of nodes during a particular round in TDMA schedule of clusters.

Ts - Time required for sensor node to transmit data frame to their corresponding cluster head.

Sensor nodes move to sleep mode after sending the data, as the sensor node are adaptable. 


\subsection{IB- LEACH}

IB-LEACH transmitted the data through gateways. With this, capacity of the nodes increases called as life time of the sensor nodes.

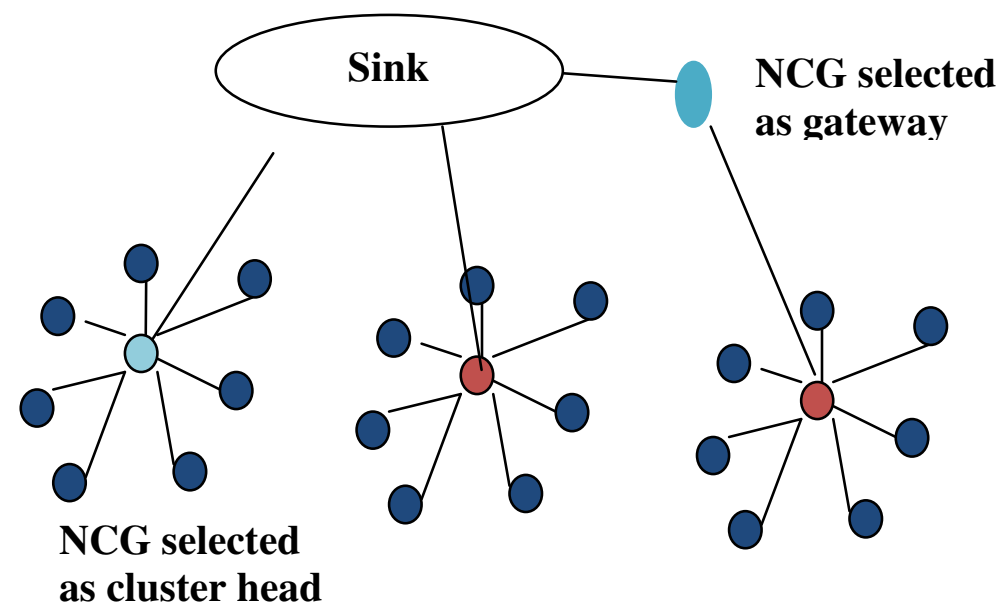

Gateway

$\bigcirc$ Cluster Head

Sensor Nodes

\section{Figure3: IB-LEACH using gateway}

A Stable Election Protocol) protocol was used to improve the LEACH protocol. It provides heterogeneity in the wireless sensor networks. This protocol provides LEACH like operation but this protocol has two different level of energy. Two tier clustering is used in SEP. in this approach, cluster head election is randomly selected and distributed based on the fraction of energy of each node assuring a uniform use of the nodes energy. SEP based on weighted election probabilities of each node to become cluster head according to their respective energy. In this some NCG are selected as cluster head and others as gateways. IB-LEACH distributes the energy load evenly among various sensor nodes. IB_LEACH is a self-organizing, adaptive clustering protocol. It does randomized rotation of the high-energy cluster-head position in such a way that various sensor nodes drain the energy from a single sensor. At any time sensor nodes can elect themselves to be a gateway. Base station confirms gateway nodes whether the node elected are suitable for gateway or not. Gateway nodes or cluster head nodes broadcast their status via advertisement message (ADV) to the other sensor nodes in the network. Non gateway nodes can also elect themselves as a cluster head with a certain probability. These cluster head nodes broadcast their status via advertisement message (ADV) to the other sensor nodes in the network.

The non-cluster-head nodes wait the cluster-head announcement from other nodes. Each sensor node determines to which cluster it wants to belong by choosing the cluster-head that requires the minimum communication energy, and send the join-request (Join-REQ) message to the chosen cluster head, and the cluster-head nodes wait for join-request message from other nodes. 


\section{PROPOSED WORK}

\subsection{Enhanced IB- LEACH}

Proposed method is the extension of existing IB-LEACH. In this method, NCG nodes are distributed based on the cluster head which reduces the energy consumption of the cluster head and thus increases the life time of the network. Results are checked through MATLAB, which shows that enhanced IB-LEACH gives better performance than the existing IB- LEACH.

\subsection{MATLAB parameters}

$100 \mathrm{~m} \times 100 \mathrm{~m}$ region of 100 sensor nodes are scattered randomly. MATLAB is used to implement the simulation. Fair comparison is done by introducing advanced energy levels to LEACH and SEP nodes with same settings as in IBLEACH protocol, so as to assess the performance of these protocols in the presence of heterogeneity.

\subsection{MATLAB Results:}

a) $\mathrm{CHs}$ are shown from Base Station and chain leader.

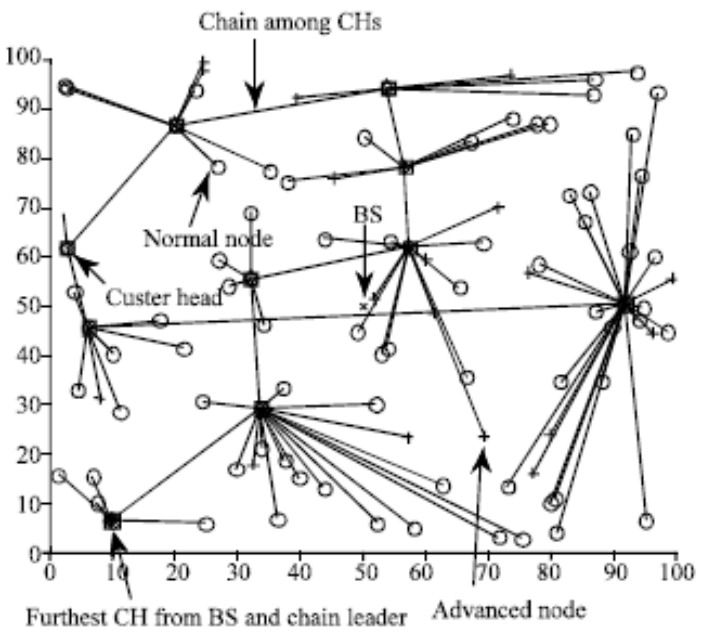

Figure 4: Base Station CHs

Normal nodes are combined together to make a cluster head.

b) Comparison is done using various different parameters as shown in graph. 
Advanced Computing: An International Journal ( ACIJ ), Vol.3, No.5, September 2012

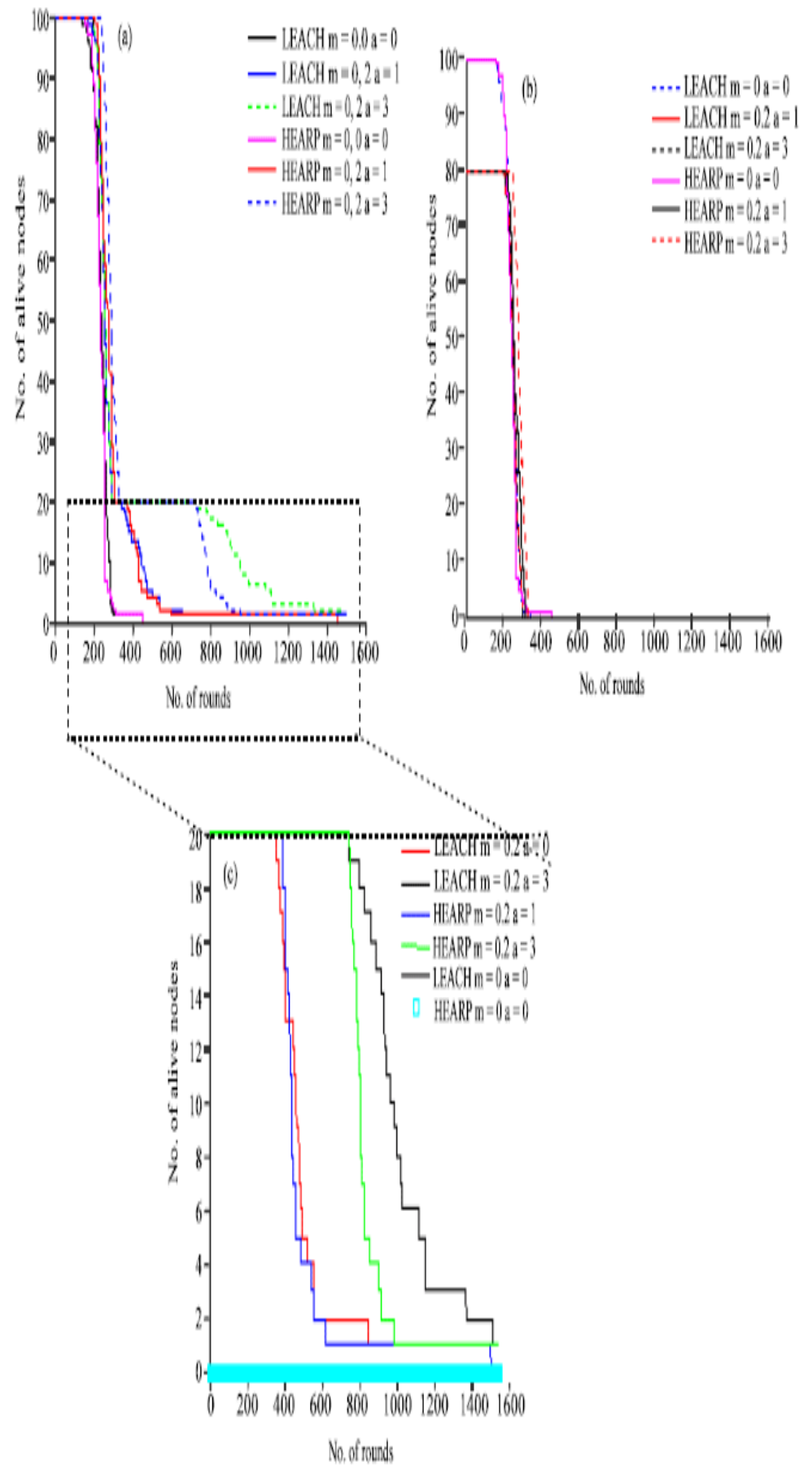

Figure 5: comparison with different data

c) Various parameters are used to show the difference between existing and enhanced IBLEACH. This graph is between numbers of alive nodes versus number of rounds. 
Advanced Computing: An International Journal ( ACIJ ), Vol.3, No.5, September 2012

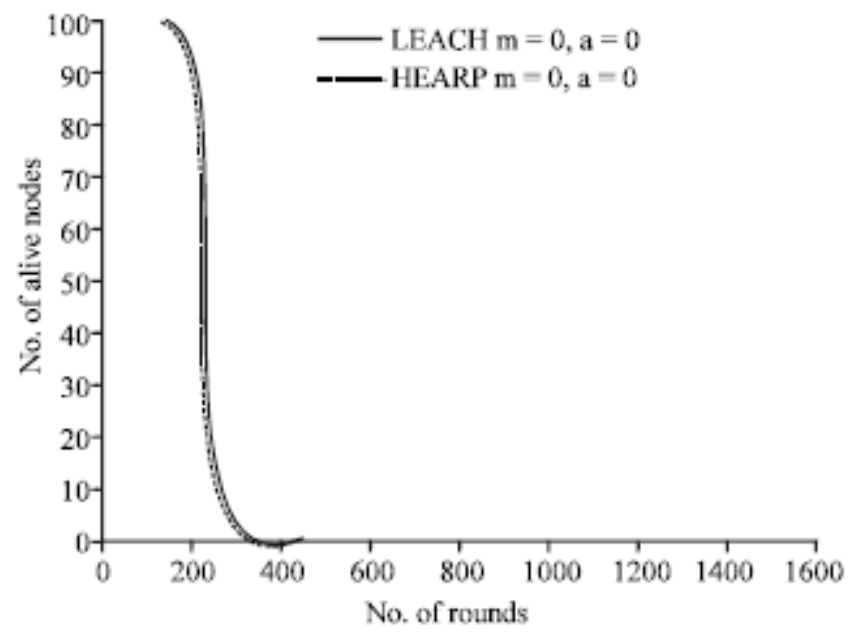

Figure 5: Alive nodes Vs. Round

d) Proposed IB-LEACH: Enhanced IB-LEACH is shown in two graphs 6(a) and 6(b) for both homogenous and heterogeneous networks.

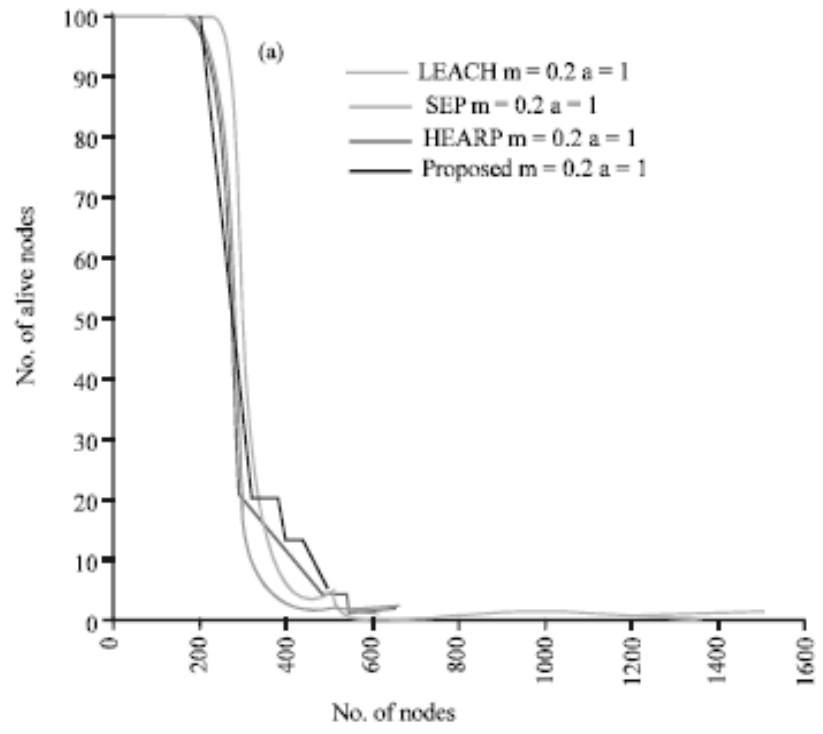

Figure 6a): Enhanced IB-LEACH 


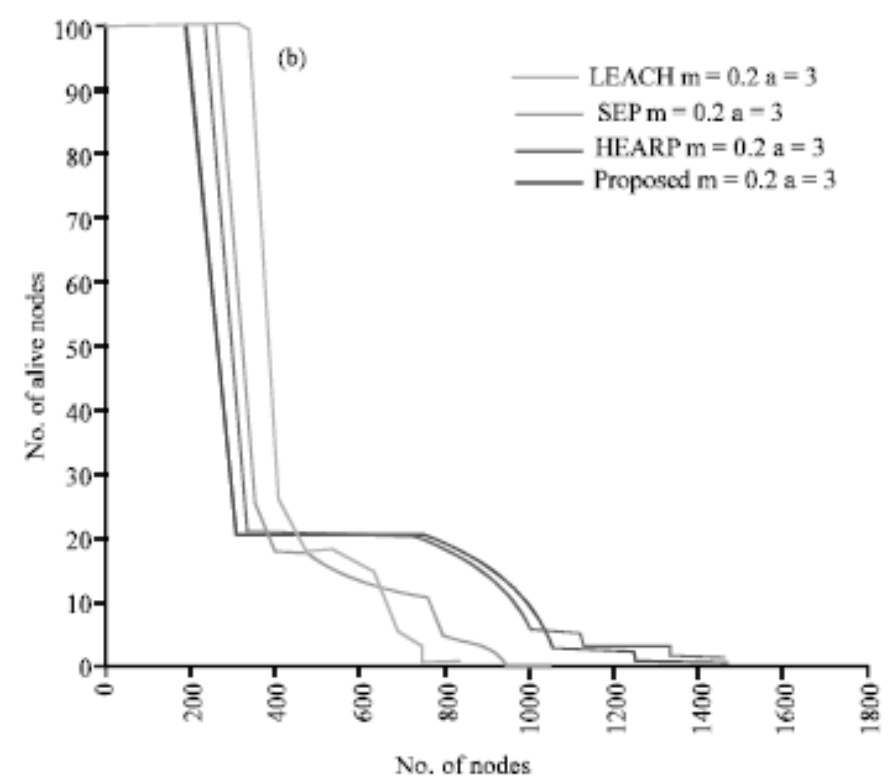

Figure 6b): Enhanced IB-LEACH

\section{CONCLUSION AND FUTURE WOK}

Wireless sensor networks consists of thousands of tiny, low cost, low power and multifunctional sensor nodes where each sensor node has very low battery life. Various energy efficient algorithms have been designed for this. LEACH uses distributed cluster formation \& randomized rotation of the cluster head to minimize the network energy consumption. This paper is proposing an algorithm which is the enhancement of existing IB-LEACH. MATLAB is used to implement the simulation. Fair comparison is done by introducing advanced energy levels to LEACH and SEP nodes with same settings as in IBLEACH protocol, so as to assess the performance of these protocols in the presence of heterogeneity. Enhanced IB-LEACH is designed for both homogenous and heterogeneous networks. The field of wireless sensor network is very challenging and recent. Various static methods and parameters can improve the performance of the network. Research can be done on choice of cluster head. Cluster head can be chosen based on the density of nodes.

\section{REFERENCES}

[1] G. Smaragdakis, I. Matta, A. Bestavros, SEP: A Stable Election Protocol for clustered heterogeneous wireless sensor networks, in: Second International Workshop on Sensor and Actor Network Protocols and Applications (SANPA 2004), 2004.

[2] W. R. Heinzelman, A. P. Chandrakasan, and H. Balakrishnan "An application-specific protocol architecture for wireless microsensor networks," IEEE Transactions on Wireless Communications, vol. 1, no. 4, pp. 660-670, October 2002.

[3] A. HosseinAlipour, D. KeyKhosravi, A.Mirzaei Somarin "New method to decrease probability of failure nodes in WSNs", (IJCNS) International Journal of Computer and Network Security,Vol. 2, No. 2, February 2010 . 
[4] V. Mhatre and C. Rosenberg, "Homogeneous vs. heterogeneous clustered sensor networks: A comparative study," in Proceedings of 2004 IEEE International Conference on Communications (ICC 2004), June 2004.

[5] I. Akyildiz, W. Su, Y. Sankarasubramaniam, and E. Cayirci, "A survey on sensor networks," IEEE Communications Magazine, vol. 40, no. 8, pp. 102-114, August 2002.

[6] I.F. Akyildiz, W.J. Su, Y. Sankarasubramaniam, E. Cayirci, Wireless sensor networks: a survey, Computer Networks 38 (2002) 393-422.

[7] K. Akkaya, M. Younis, A survey on routing protocols for wireless sensor networks, Ad Hoc Networks 3 (3) (2005) 325-349.

[8] Gaurav Gupta, Mohamed Younis "Fault-Tolerant Clustering of Wireless Sensor Networks" 2003 IEEE

[9] Ameer Ahmed Abbasi and Mohamed Younis: A survey on clustering algorithms for wireless sensor networks,

\section{Authors}

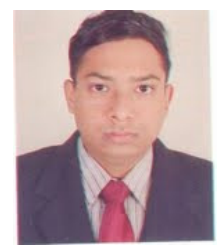

Yogesh Kumar is doing part time M.TECH from India's most reputed Institute Guru Nanak Dev Engg. College Ludhiana, situated in Punjab. He is also working as an Assistant Professor in ECE Department in Ludhiana College of Engg. And Technology, Punjab. He had total 8 years of experience in Teaching and Industry. His area of interest is Optical Communication and Wireless Sensor Networks.

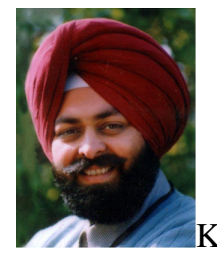

Kanwaljit Singh is working as an Associate Professor in India's most reputed Institute Guru Nanak Dev Engg. College Ludhiana, situated in Punjab. He had done his M.E. in Electronics in 1992 and having 25 years of teaching experience. His area of interest is Optical Communications, Microprocessors. 